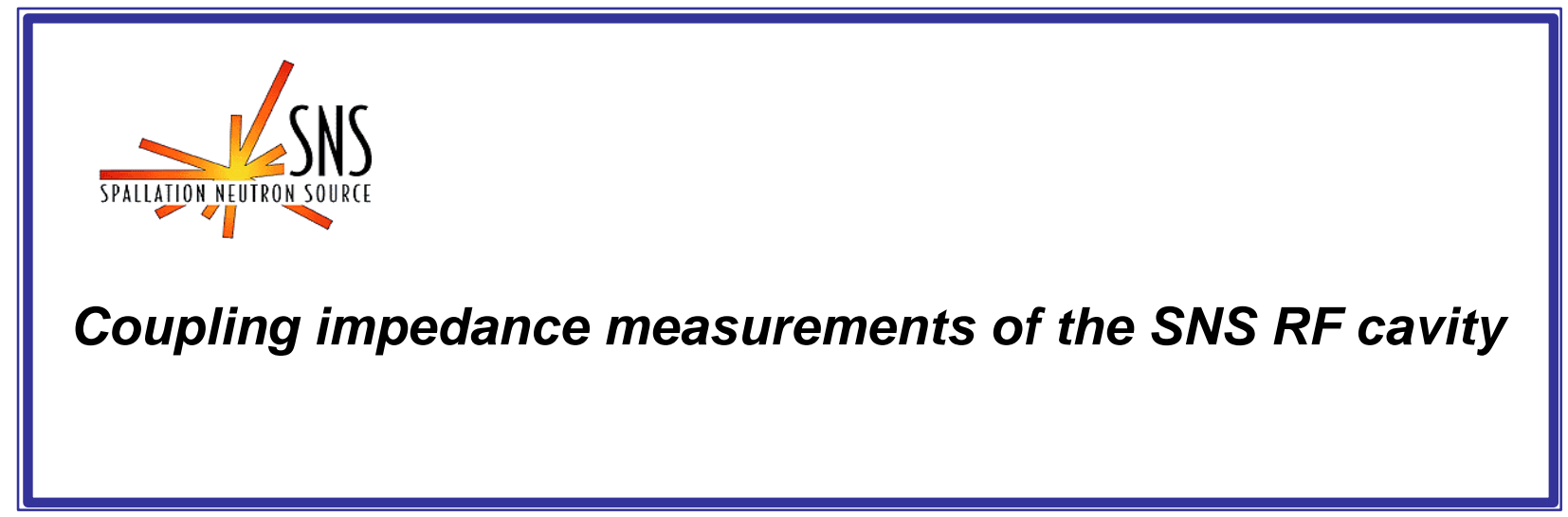

BNL/SNS TECHNICAL NOTE

NO. 115

D. Davino, H. Hahn, M. Blaskiewicz

August 27, 2002

COLLIDER-ACCELERATOR DEPARTMENT BROOKHAVEN NATIONAL LABORATORY UPTON, NEW YORK 11973 


\title{
Coupling impedance measurements of the SNS RF cavity
}

\author{
D. Davino, H. Hahn and M. Blaskiewicz \\ Brookhaven National Laboratory, Upton, NY 11973 USA
}

\begin{abstract}
In this report the bench measurements on the SNS RF cavity are presented. Both longitudinal and transverse impedance measurements have been performed and contributions to the SNS ring impedance budget are provided. A solution to damp a relevant transverse resonance is demonstrated.
\end{abstract}

\section{Introduction}

The transverse coupling impedance of a circular accelerator or storage ring can cause beam instabilities and must be carefully monitored in order to keep its value within the impedance budget. The success of the SNS (Spallation Neutron Source) will largely depend on reducing the transverse coupling impedance of the accumulator ring.

The SNS RF system is composed by four cavities, three of them are driven on the first beam harmonic and one is driven on the second one. Harmonic numbers imply RF frequencies of $f_{1}=$ $1.058 \mathrm{MHz}$ and $f_{2}=2.116 \mathrm{MHz}$. Each cavity is composed of two RF gaps and the power amplifier drives them in parallel by means of side bus-bars. The first harmonic cavities $(h=1)$ are designed for a gap voltage of $V_{g}=10 \mathrm{kV}$ and the second harmonic cavities $(h=2)$ are identical but for less gap capacitance. The gap capacitance for $h=1$ cavities is obtained by adding four capacitors of 750 $\mathrm{pF}$ in parallel, assembled as it is shown in Fig. 1 right, the total being $3 \mathrm{nF}$. The $h=2$ cavity is equal but for only one $750 \mathrm{pF}$ gap capacitance. The inductance is supplied by coaxial stacks of Philips 4M2 ferrite rings. There are 21 rings per gap and each ring is $2.72 \mathrm{~cm}$ thick. The rings have inner and outer diameters of $25 \mathrm{~cm}$ and $50 \mathrm{~cm}$, respectively. The total length of the device is about 2.7 $\mathrm{m}$. More details can be found in [1].

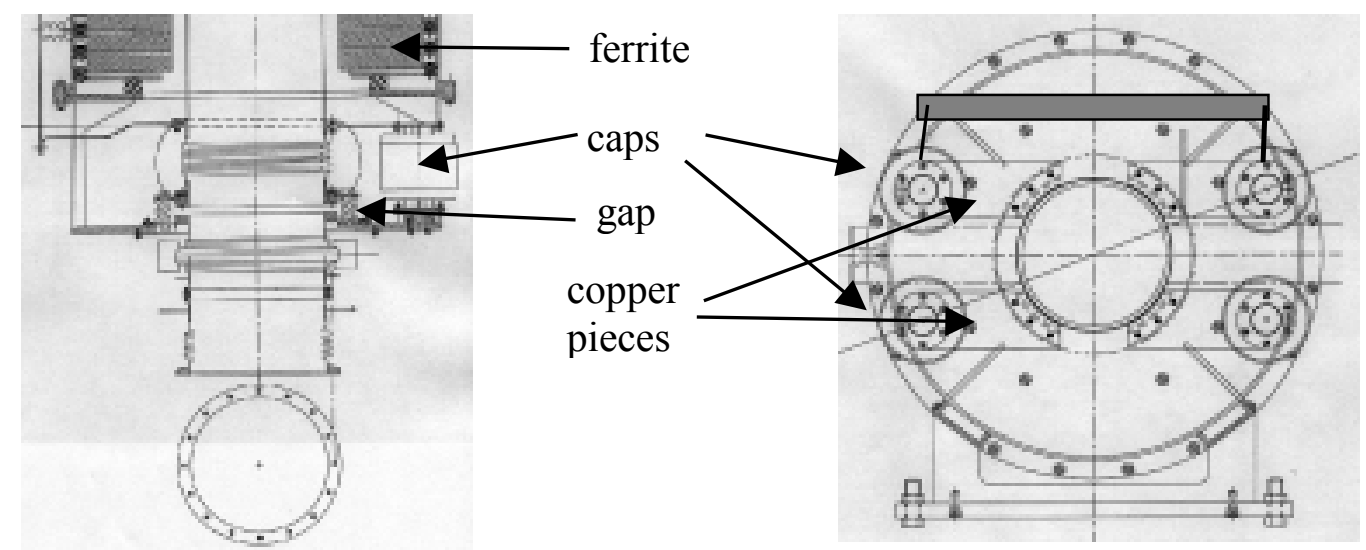

Fig. 1: Drawings of the cavity end seen from the top (left) and of the transverse section (right). Also shown is a sketch of a $40 \Omega$ carborundum rod to be added. 
Longitudinal and transverse bench measurements on the RF cavity were made using the standard method [2]; in which a single wire, or a twin-wire "Lecher" line, is inserted into the cavity. In the next section, the longitudinal impedance measurements are presented. In the third section, the transverse impedance measurements are presented and commented.

\section{Longitudinal impedance measurements}

For the longitudinal impedance measurements, the forward transmission coefficients $\mathrm{S}_{21}$ of the "Device Under Test" and of a coaxial reference line is interpreted according to

$$
Z_{\|}^{D U T}=-2 Z_{L} \ln \left(S_{21}^{D U T} / S_{21}^{R E F}\right)
$$

with $Z_{L}$ the characteristic impedance of the coaxial line. It is worth noting that the length of the cavity, and therefore of the reference tube and wire, is comparable to the wavelengths of the measured frequency range. But, thanks to the end matching resistors, this does not produce longitudinal resonances.

In Fig. 2 left, the longitudinal coupling impedance of the cavity, set for the first harmonic, is shown. In Fig. 2 right the second harmonic case is shown; this case is obtained by removing three, out of four, lumped capacitance in parallel to the cavity gaps. The main resonance is at $846 \mathrm{MHz}$ for the first harmonic case, and not at the correct $1.058 \mathrm{MHz}$, because of the absence of the ferrite bias current.
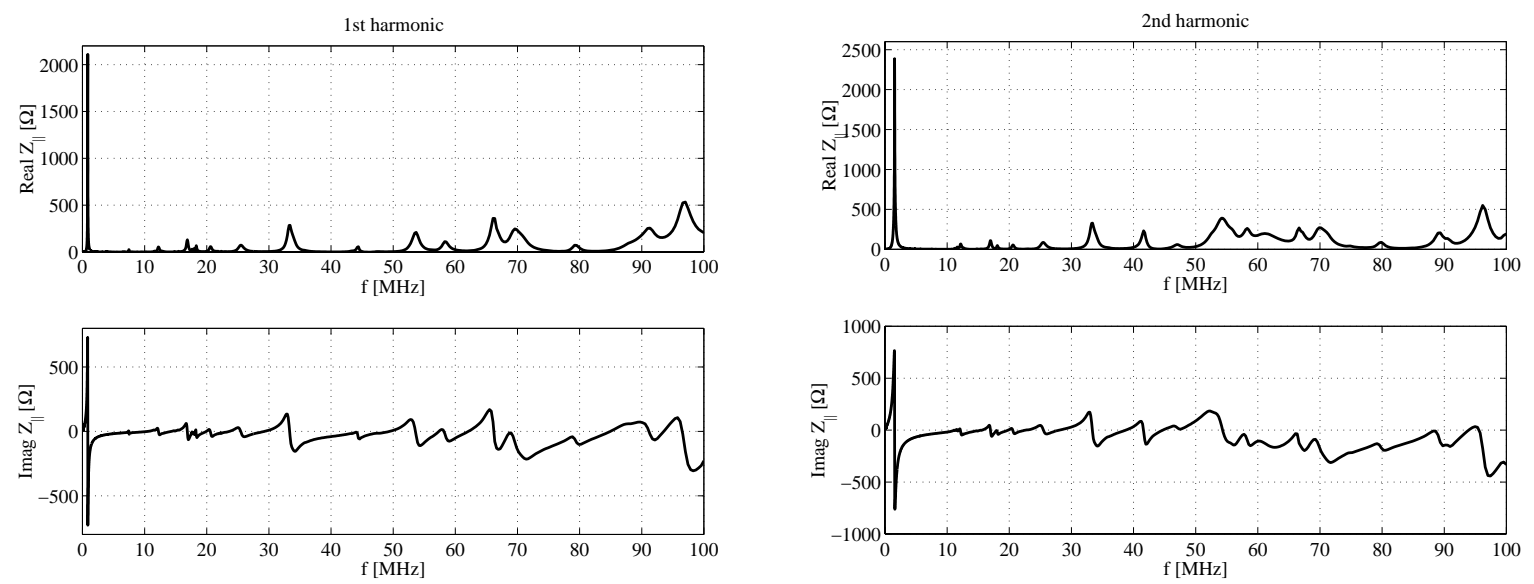

Fig. 2. Longitudinal impedances of the cavity set for the first harmonic (left) and for the second harmonic (right).

In Fig. 3, the effect on the longitudinal impedances by shorting the gaps with a safety switch is shown. The response of the first and second harmonic cavity is the same. One can see that the main resonance is shifted by the same amount when either gap is shorted implying that the cavity gaps are symmetric and in parallel, without any bus-bar induced phase shift. When both gaps are shorted the main resonance is still present but the shift is larger.

Shorting the gaps causes only a change in the shunt impedance of some of the higher order modes, but none of them has a frequency shift. 

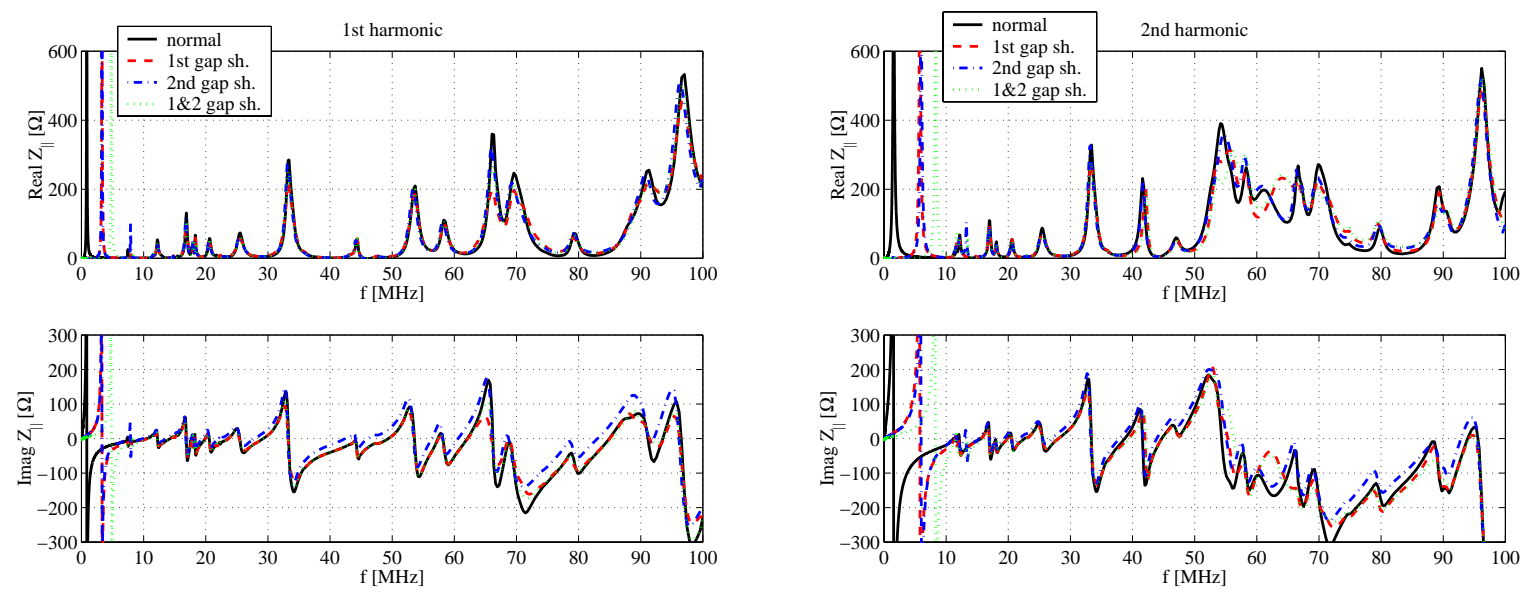

Fig. 3. Longitudinal impedances of the cavities set for the first (left) and second (right) harmonics, short-circuited gaps.

Fig. 4 and 5 show the longitudinal impedance normalized to the mode number $n=f / 1.058 \mathrm{MHz}$. The high order modes for the first harmonic cavity have:

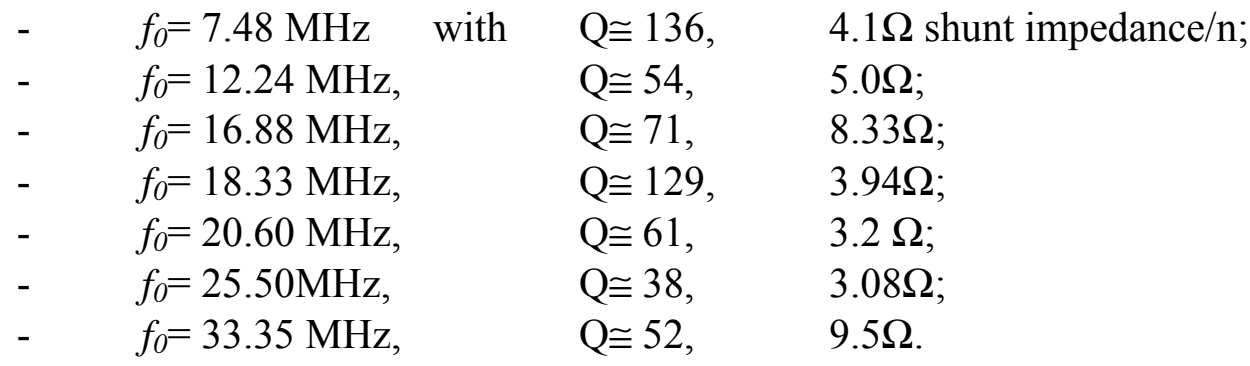
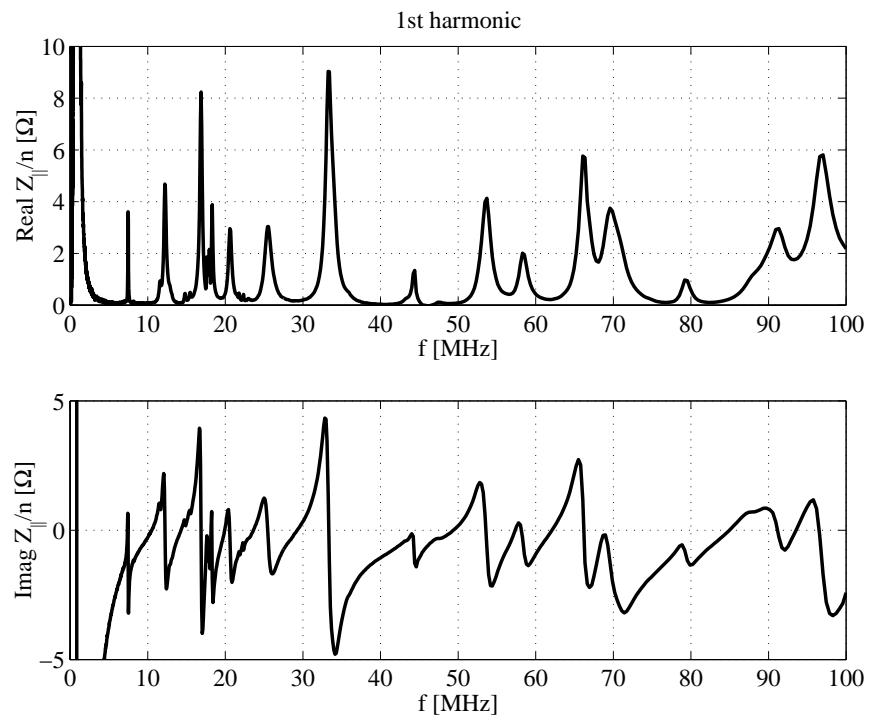

Fig. 4. Longitudinal impedances of the cavity set for the first harmonic, normalized to the mode number $\mathrm{n}=\mathrm{f} / 1.058 \mathrm{MHz}$. 

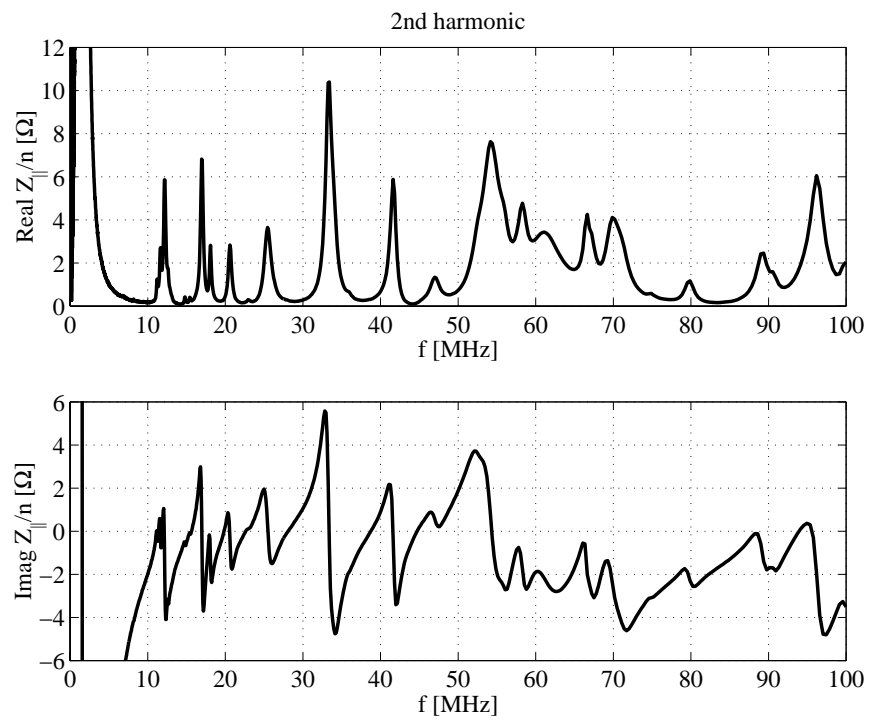

Fig. 5. Longitudinal impedances of the cavity set on the second harmonic, normalized to the mode number $\mathrm{n}=\mathrm{f} / 1.058 \mathrm{MHz}$.

\section{Transverse coupling impedance measurements}

The transverse coupling impedance has been measured by means of a homemade twin-wire cable. In analogy to the longitudinal measurement, the transverse impedance follows from:

$$
Z_{x, y}=\frac{c Z^{D U T}}{\omega \Delta^{2}}=-2 \frac{c Z_{L}}{\omega \Delta^{2}} \ln \left(S_{21}^{D U T} / S_{21}^{R E F}\right)
$$

with $\Delta=41 \mathrm{~mm}$ being the spacing of the two wire and $Z_{L}=215 \Omega$, the characteristic impedance of the twin-wire line.

At first, the cavity was measured in the frequency range $100 \mathrm{kHz}-100 \mathrm{MHz}$, by using as a reference the measurements of the twin-wire in air, without any reference beam pipe. This was the probable cause of a ratio between the transmission coefficients larger than the unity, leading to conceptually wrong impedance with a negative real part (Fig. 6). Moreover, a sort of amplitude modulation was present along the frequency. In spite of that, a resonant mode was clearly visible at about $17.6 \mathrm{MHz}$ when the cavity was in the first harmonic configuration and the cable plane was horizontal. No significant impedance was measured with the cable in the vertical direction. The second harmonic configuration did not give that mode in the measured frequency range. 

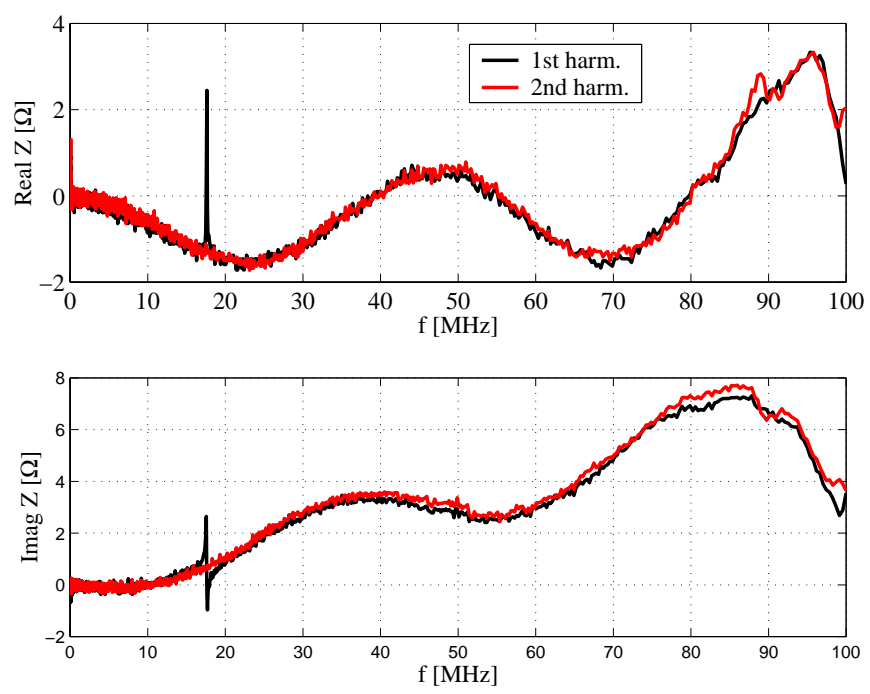

Fig. 6. Measured impedance, $Z^{D U T}$, of the two configurations, by using as a reference the measurements of the twin-wire in air, without any reference beam pipe.

The fact that only one mode was present in the impedance measurements and that it was disappearing by measuring the cavity without the gaps capacitors suggested to use this measurement as reference. As a consequence, the impedance was again non-negative. In order to better visualize the resonance, the frequency range was restricted to $15-40 \mathrm{MHz}$.

In Fig. 7 the measured transverse impedance of the cavity is shown. The solid (black) curve shows two resonances, associated with the two gaps. The higher one has a peak of about $6 \mathrm{k} \Omega / \mathrm{m}$ at 17.65 MHz and the lower one has a peak of about $2 \mathrm{k} \Omega / \mathrm{m}$ at $18.22 \mathrm{MHz}$. The dashed (red) curve shows the transverse impedance after placing four $40 \Omega$ carborundum rods (glow-bars) on top and bottom of each gap, connected to the capacitors through alligators contacts. It is apparent that the glow-bars damp the resonances by a factor 2 .
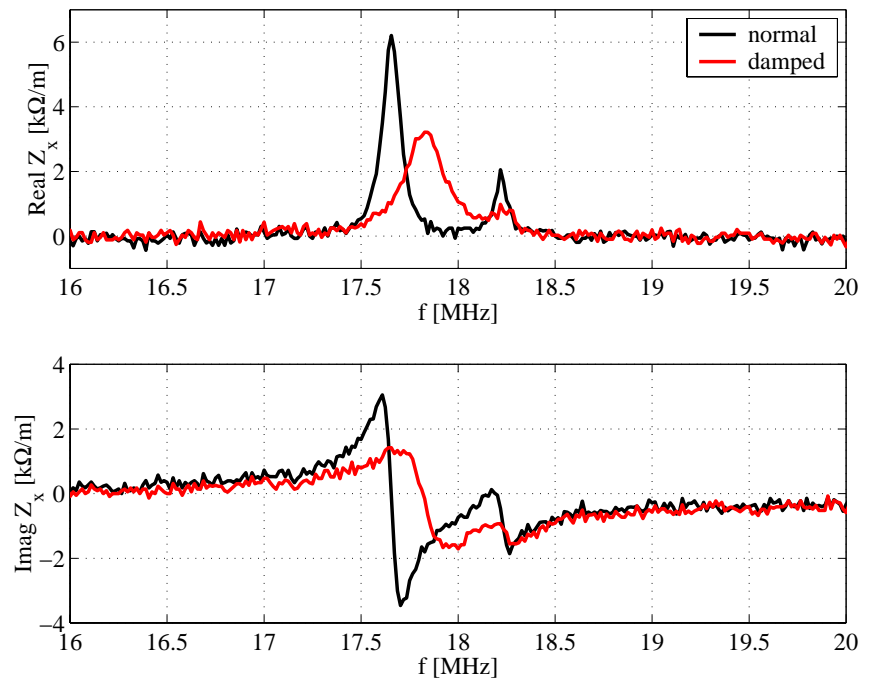

Fig. 7. Measured transverse impedance of the cavity with two gaps set for the first harmonic. 
An equivalent analytical expression for the main resonance damped with glow-bars is:

$$
Z_{x}=\frac{c}{\omega} R_{s}\left[1+j Q\left(f / f_{0}-f_{0} / f\right)\right]^{-1}
$$

where $R_{S}=1190 \Omega / \mathrm{m}^{2}, Q=75$ and $f_{0}=17.82 \mathrm{MHz}$. This leads to the impedance shown in Fig. 8:
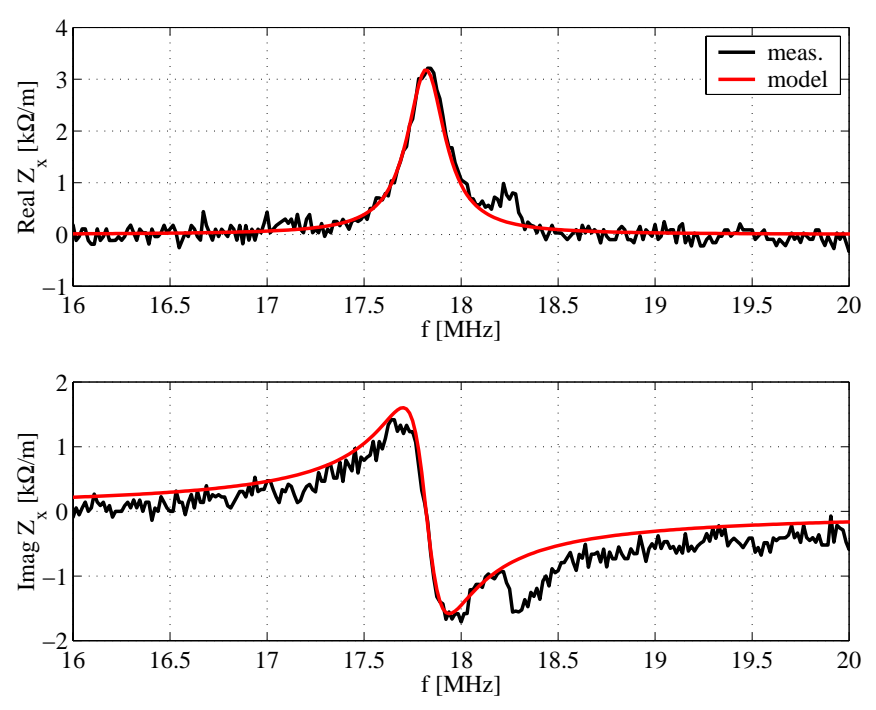

Fig. 8. Measured and equivalent transverse impedance of the cavity with glow-bars.

In order to obtain the total contribution to the impedance budget, one has to multiply eq. (3) by a factor 3, which is the SNS baseline-number of first harmonic cavities. However, it is worth noting that this value is horizontal and not additive to the vertical extraction kickers impedance.

\section{Other measurements}

Several capacitors arrangements were measured on the cavity, and the more interesting measurements are commented in this section.

In Fig. 9 is shown the transverse impedances of the cavity with only two $750 \mathrm{pF}$ capacitors placed at the bottom of one gap. The black curve indicates that one resonant mode is still present, at about 19.7 $\mathrm{MHz}$ and with a peak of about $2 \mathrm{k} \Omega / \mathrm{m}$. As stated in the previous section, the use of a glow-bar connecting the capacitors damps the resonance. It is important to note that the glow-bar has to be placed near the gap side (red curve) in order to be more effective. Indeed, the glow-bar placed near the ground side of the gap (green curve) provides less damping. The damping factor is much larger here than for the baseline configuration.

The capacitance plays an important role in the resonance, together with the small inductance $(\sim 0.02 \mu \mathrm{H})$ of the copper pieces connecting the capacitors to the gap. It seems that the resonance is present only if the capacitors are placed on both sides, left and right, of either gap. The gap with 
only one capacitor (second harmonic configuration) does not have a resonance, and even two capacitors on one side of the gap do not produce a resonance.
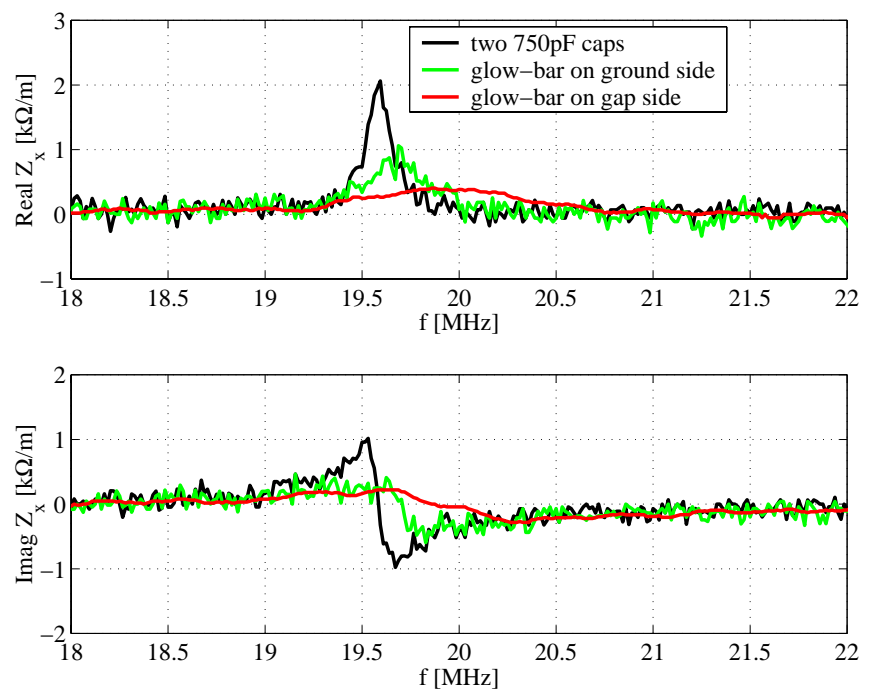

Fig. 9. Measured transverse impedance of the cavity with two gaps set for the first harmonic.

Replacing the $750 \mathrm{pF}$ with $300 \mathrm{pF}$ capacitors of the same company and type shifts the resonance to about $31.3 \mathrm{MHz}$. Fig. 10 (black curve) shows the case when at the bottom of one gap there are two 750 caps, damped by a glow-bar; and at the top of the same gap there are two $300 \mathrm{pF}$. The red curve shows that it is possible to damp the $31.3 \mathrm{MHz}$ resonance with a glow-bar as well. Moreover, this measurement makes clear that the top and bottom part of the gap act almost independently, each one developing a resonance.
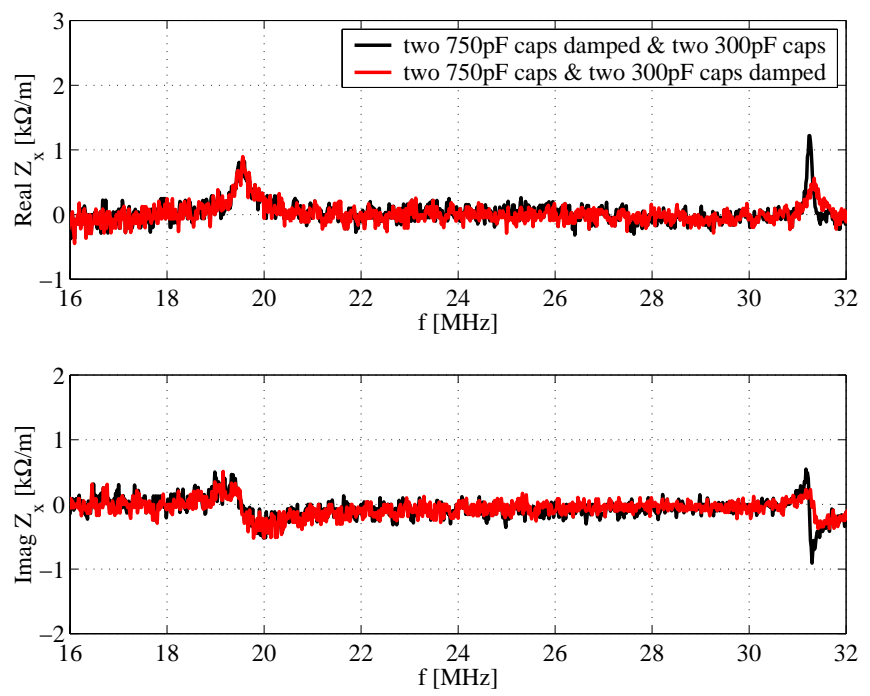

Fig. 10. Measured transverse impedance of the cavity with $750 \mathrm{pF}$ and $300 \mathrm{pF}$ capacitors.

In order to disprove the conjecture that the resonance is caused by the particular baseline capacitors type, they were replaced by two high-frequency $375 \mathrm{pF}$ capacitors. Fig. 11 shows that a resonance is still present. This confirms that the resonance is not connected with the particular 
baseline capacitor type. The frequency shift does not agree exactly with the capacitances ratio, suggesting that another capacitive contribution is given by the gap itself.
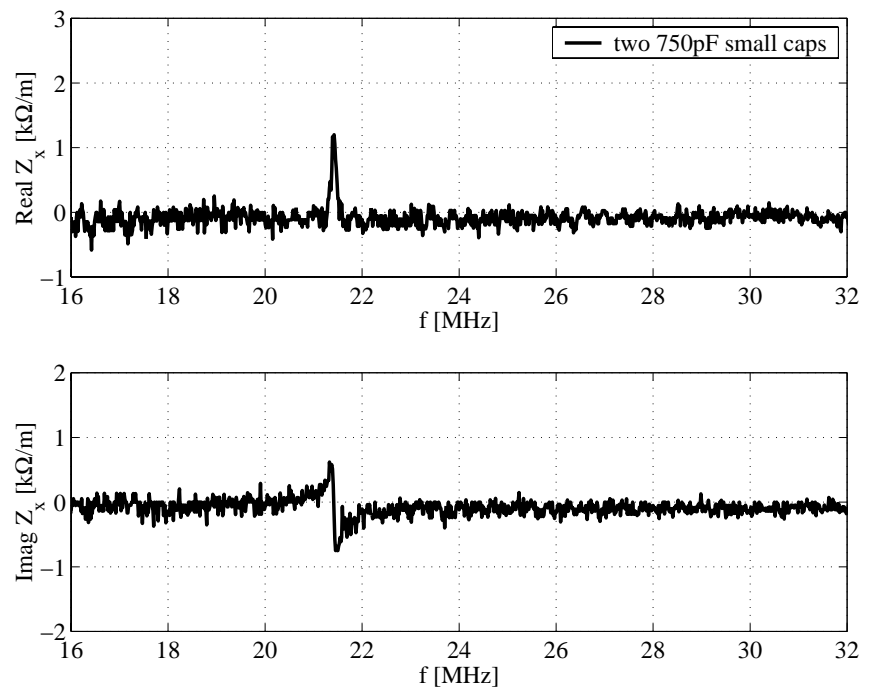

Fig. 11. Measured transverse impedance of the cavity with $375 \mathrm{pF}$ high-frequency capacitors.

\section{Acknowledgements}

The authors would like to thank A. Zaltsman for the use of the cavity. Damping the transverse mode with glow-bars across the capacitors was suggested by J.M. Brennan.

\section{References}

[1] M. Blaskiewicz, J.M. Brennan, J. Brodowski, J. Delong, M. Meth, K. Smith, A. Zaltsman, RF System for the SNS Accumulator Ring, Proc. 2001 PAC, Chicago, IL, p. 490.

[2] F. Caspers, in Handbook of Accelerator Physics and Engineering, ed. A.W. Chao and M. Tigner, (World Scientific, Singapore, 1998), p. 571. 